\title{
Des amphores et des hommes
}

\section{Chronique 2014}

\section{Fanette Laubenheimer}

DANS Dialogues D'Histoire ANCIENNe 2014/1 (40/1), PAGES 213 À 238

Éditions PREsses Universitaires de FranCHE-COMTÉ

\section{ISSN $0755-7256$}

ISBN 9782848674872

DOI 10.3917/dha.401.0213

Article disponible en ligne à l'adresse

https://www.cairn.info/revue-dialogues-d-histoire-ancienne-2014-1-page-213.htm

Découvrir le sommaire de ce numéro, suivre la revue par email, s'abonner...

Flashez ce QR Code pour accéder à la page de ce numéro sur Cairn.info.

Distribution électronique Cairn.info pour Presses universitaires de Franche-Comté.

La reproduction ou représentation de cet article, notamment par photocopie, n'est autorisée que dans les limites des conditions générales d'utilisation du site ou, le cas échéant, des conditions générales de la licence souscrite par votre établissement. Toute autre reproduction ou représentation, en tout ou partie, sous quelque forme et de quelque manière que ce soit, est interdite sauf accord préalable et écrit de l'éditeur, en dehors des cas prévus par la législation en vigueur en France. Il est précisé que son stockage dans une base de données est également interdit. 


\section{Des amphores et des hommes* \\ Chronique 2014}

Fanette LAUbENHEIMER

*Abrégé en DADH

Les lecteurs de cette chronique (non exhaustive) sont invités à l'enrichir en signalant articles et publications à Fanette Laubenheimer, UMR 7041 Archéologies et Sciences de l'Antiquité, Équipe Archéologie de la Gaule, Structures Économiques et Sociales, Maison de l'Archéologie et de l'Ethnologie René Ginouvès, 21 Allée de l'Université, 92023 Nanterre cedex,E.mail:fanette.laubenheimer@mae.u-paris10.fr

\section{GÉNÉRALITÉS}

1, A. et J.-P. Joncheray, $\grave{A}$ la découverte de l'amphore, classification et histoire, Hyères, 2013, deux infatigables chercheurs d'épaves sur la côte provençale, depuis le début des années 70 , livrent un catalogue photographique remarquable des amphores entières des 78 types qu'ils ont pu rencontrer, des premières étrusques ou marseillaises aux amphores sarrasines et tardives, avec une pédagogie qui sera fort utile aux jeunes chercheurs et au grand public.

\section{LES AMPHORES JUSQU' AU II ${ }^{\mathrm{E}}$ SIĖCLE AVANT NOTRE ÈRE}

\section{En Méditerranée orientale}

2, Y. Garlan, « Les timbres amphoriques en Grèce ancienne. Nouvelles questions. Nouvelles méthodes. Nouveaux résultats », Journal des Savants, Juillet-décembre, 2013, p. 203-270, dans ce bel article de quelque 70 pages, Yvon Garlan nous livre avec recul son 
regard global sur ce sujet qui l'a passionné tant d'années. De la présentation d'un matériel fabuleux, autour de 400000 timbres amphoriques grecs, en passant par l'historiographie du sujet depuis le XIX ${ }^{\mathrm{e}}$ siècle (timbre fiscal, certificat de capacité et de garantie, datation, commerce et controverses sur la nature du timbrage), il nous conduit au renouveau de l'amphorologie grecque avec la découverte des ateliers à partir de la fin des années 70, à laquelle il a largement participé, et à l'analyse physicochimique des pâtes. L'étude de la place des fabricants dans le timbrage grec et l'interprétation finale de ce timbrage, des questions complexes, arrivent aux conclusions suivantes : il s' agit d'un timbrage d'origine publique et de caractère administratif dont la finalité reste encore indécise. La porte est largement ouverte à de nouvelles recherches non seulement sur le timbrage, mais aussi sur la structure même des ateliers encore mal connus.

3, J. Remesal Rodríguez, V. Porcheddu, M. Garcia Sanchez, « Sodales Adiuvate! Il contributo dell'informatica al progresso dell'epigrafia anforica greca », Epigraphica, LXXV, 1-2, 2013, p. 309-335, présentent un historique des recherches sur les timbres amphoriques grecs, véritables sources historiques, et la constitution plus ou moins récentes des diverses bases de données informatisées concernant leur grande quantité. Au-delà de simples corpus de timbres, les auteurs plaident en faveur du rôle essentiel des amphores grecques timbrées pour l'histoire sociale et pour l'histoire économique. Ils insistent sur le rôle indispensable de l'informatique dans ces domaines et invitent les chercheurs à une collaboration globale au sein de la banque de données créée par le CEIPAC.

4, P. Monsieur, B. Overlaet, S. A. Jasim, E. Yousif, E. Haerinck, « Rhodians amphora stamps found in Mleiha (Sharjah, UAE): old and recent finds », Arabian archaeology and epigraphy, 2013, p. 208-223, dix-huit timbres rhodiens ont été trouvés dans des contextes funéraires à Mleiha, à $45 \mathrm{~km}$ à l'ouest du golfe d'Oman. Onze d'entre eux datent de la deuxième moitié du $\mathrm{III}^{\mathrm{e}}$ siècle et de la première moitié du $\mathrm{II}^{\mathrm{e}}$ siècle av. J.-C. À cette période, Mleiha est le seul site actuellement connu dans la péninsule d'Oman.

\section{En Méditerranée occidentale}

5, A. Ribera i Lacomba (éd.), Manuel de cerámica romana. Del mundo Helenistico al Imperio Romano, Madrid 2013, issu d'un cours de formation permanente pour les archéologues, cet ouvrage présente notamment un chapitre sur les amphores: G. Pascual Berlanga, A. Ribera i Lacomba, «El materiel más aprceciado pour los antiguos. Las ánforas », p. 215-289, présentent les amphores préromaines étrusques, grecques d'Orient et d'Occident et celles de Grande Grèce. Pour le monde romain 
républicain, ils passent en revue les productions d'Italie, gréco-italiques, Dressel 1, Lamboglia 2, amphores de l'Adriatique et de Brindisi. Après un bref coup d'œil sur les amphores vinaires orientales, les auteurs présentent avec plus de détail les amphores puniques à vin, huile et poisson et leurs divers lieux de production.

\section{- En Italie}

6, G. Bron, « Les amphores du dépôt du secteur 4 de l'Incoronata (Basilicate) : essai de typochronologie et contextuel d'une classe céramique du VII ${ }^{\mathrm{e}}$ siècle av. J.-C. », Mélanges de l'École Française de Rome, 123-2, 2011, p. 467-504, dans un site d'habitat, parmi les amphores des trois premiers quarts du VII ${ }^{\mathrm{e}}$ siècle av. notre ère, les productions corinthiennes dominent, les gréco-orientales et attiques sont en nombre bien inférieur.

7, F. Cibecchini, C. Capelli, « Nuovi dati archeologici e archeometrici sulle anfore greco-italiche i relitti di III secolo del Mediterraneo occidentale e la possibilità di una nuova classificazione », dans F. Olmer (éd.), Itinéraires des vins romains en Gaule, Monographies d'Archéologie Méditerranéenne n5, Lattes, 2013, p. 423-451, reprennent l'épineux problème de l'origine et de la classification des gréco-italiques. Dès le début $\mathrm{du} \mathrm{III}^{\mathrm{e}}$ siècle avant notre ère, des ateliers très dispersés et encore mal connus produisent des formes similaires, de la côte adriatique à la tyrrhénienne en passant par la Sicile. Les auteurs choisissent donc de travailler à partir du matériel d'une quinzaine d'épaves du III ${ }^{\mathrm{e}}$ siècle av. J.-C. Leur but est de donner une nouvelle classification fondée sur la typologie, la chronologie, l'épigraphie et l'archéométrie. Des analyses physicochimiques définissent cinq groupes, $\mathrm{A}$ à $\mathrm{E}$, auxquels est attribuée une localisation géographique. Le classement typologique reprend la séquence proposée par Vandermerch : groupes III à $\mathrm{V}$ en indiquant les caractéristiques morphologiques, la chronologie, les zones de production possibles et l'épigraphie, voire la diffusion. La relation entre les groupes physicochimiques et les groupes typologiques n'est pas toujours très claire, mais il s'agit d'un vaste travail encore en cours qui déjà apporte des résultats intéressants.

\section{- En Gaule}

8, F. Saccheti, J.-C. Sourrisseau, « Sur les importations d'amphores en contextes hallstattiens : regards croisés depuis le Midi de la Gaule et le bassin nord-adriatique », dans A. Colin, F. Verdin (éds), L'âge du fer en Aquitaine et sur ses marges. Mobilité des hommes, diffusion des idées; circulation des biens dans l'espace européen à l'âge du fer, Aquitania, Bordeaux, 2013, p. 643-664, la nouveauté des cartes de distribution des amphores grecques dans ces deux régions semble confirmer l'existence simultanée de 
relations rhodaniennes et transalpines dans cette phase chronologique correspondant au « phénomène princier ».

9, R. Roure, «Les circulations entre Languedoc et Berry au $\mathrm{V}^{\mathrm{e}}$ siècle avant notre ère : le champ des possibles (hypothèses et pistes d'étude) », dans F. Olmer (éd.), Itinéraires des vins romains en Gaule, Monographies d'Archéologie Méditerranéenne n5, Lattes, 2013, p. 7-14, contre l'idée du rôle dominateur de Marseille pour les exportations de vin et de vaisselle vers l'intérieur du pays, l'auteur suggère la participation active des sociétés méridionales dans ce commerce, notamment suivant un trajet Languedoc oriental, Massif central, Bourges. Il ne s'agit que d'une piste de recherche qui aura encore besoin d'être étayée.

10, P. Séjalon, « Les faciès amphoriques précoces en Languedoc occidental », dans F. Olmer (éd.), Itinéraires des vins romains en Gaule, Monographies d'Archéologie Méditerranéenne n5, Lattes, 2013, p. 15-22, l'analyse des amphores gréco-italiques en Languedoc occidental montre une diffusion bien documentée le long de l'axe de l'Aude dès la fin du IV ${ }^{\mathrm{e}}$ siècle et au III ${ }^{\mathrm{e}}$ siècle. Le commerce serait contrôlé par les Ibères dès le III ${ }^{\mathrm{e}}$ siècle. Une forte croissance des importations se marque au milieu du III $^{\mathrm{e}}$ siècle, parallèlement à la politique d'annexion des vignobles de Grande Grèce par Rome et au développement de nouveaux vignobles dans le Latium et la Campanie. Ce commerce périclite autour de 250/225.

\section{AMPHORES RÉPUBLICAINES ET IMPÉRIALES}

\section{En Méditerranée occidentale}

\section{- En Italie}

11, M. Mongardi, « Anfore con titulipicti dallo scavo di Modena, Parco Novi Sad: alcune osservazioni », Epigraphica, LXXV, 1-2, 2013, p. 429-438, étudie une douzaine de marques peintes bien conservées sur des amphores à salaison de poisson de Bétique (Beltran IIA, Dr. 8 et Dr. 12), parmi la soixantaine d'amphores de ces types appartenant à ce dépotoir ou un vide sanitaire de Modène, composé de près d'un millier d'amphores et daté de la deuxième moitié du I ${ }^{\mathrm{er}}$ siècle et de la première moitié du $\mathrm{II}^{\mathrm{e}}$ siècle, plusieurs amphores appartiennent à la société familiale des Quinti Caecilii qui commercialise non seulement des salaisons mais aussi de l'huile de Bétique. L'auteur souligne l'absence de standardisation dans les marques peintes sur les amphores à poisson de Bétique qui, 
contrairement aux amphores à huile annonaire, faisaient partie de circuits commerciaux privés.

12, E. Mongardi, « Le anfore dello scavo del parco Novi Sad a Modena (Italia): considerazioni su alcuni bolli inediti », dans SFECAG, Actes du Congrès d'Amiens 2013, p. 459-464, toujours à propos du millier d'amphores de la fouille du Parc Novi Sad à Modène, E. M. présente ici quelques uns des 138 timbres recueillis : 11 timbres sur Dr. 6A de Padanie au nom d'une femme, Numisia L.f. Firmilla, 5 timbres VOLC sur Dr. 6A, d'origine picénienne vraisemblablement, et une association inédite de timbres au génitif, RVBRI et CADMI sur les deux anses d'une Dr. 6A.

13, S. Menchelli, C. Capelli et al., Nuove scoperte d'ateliers di anfore repubblicane nell'Etruria settentrionale costiera, dans F. Olmer (éd.), Itinéraires des vins romains en Gaule, Monographies d'Archéologie Méditerranéenne n5, Lattes, 2013, p. 471-478, poursuivant les recherches d'ateliers dans l'Étrurie du nord, les auteurs mettent en lumière dans la zone de Portus Pisanus. L'officine de Ca Lo Spelli produit des amphores anépigraphes Dr. 1A, et intermédiaires D 1A/B, mais surtout des Dr. 1B. Les analyses minéralogiques et pétrographiques permettent de tracer un commerce d'exportation vers le nord, à Avenches et à Augst.

14, L. Benquet, D. Vitali, F. Laubenheimer, « Nouvelles données sur l'atelier d'amphores d'Albinia (Ortobello, Italie) : campagnes de fouilles 2003-2006 », dans F. Olmer (éd.), Itinéraires des vins romains en Gaule, Monographies d'Archéologie Méditerranéenne ${ }^{\circ} 5$, Lattes, 2013, p. 513-529, les remarquables structures de cuisson de ce vaste atelier sont mises en relation avec la production d'amphores dont la typologie permet d'avancer une chronologie fixée entre la deuxième moitié du II $^{\mathrm{e}}$ siècle et le $\mathrm{I}^{\mathrm{er}}$ siècle av. J.-C. pour les grands fours 1 et 2 , les mieux conservés. La production majoritaire est constituée de Dr. 1B, les Dr. 1A et Dr. 1C sont plus rares. Certains timbres sont communs aux deux premières formes. Une pièce enterrée, de fonction encore mal définie, a fourni une majorité de Dr. 1C. Un drainage spectaculaire de quelque de $200 \mathrm{~m}^{2}$ a réutilisé de l'ordre de 400 à 500 amphores défectueuses Dr. 1B et de rares amphores ovoïdes. La dernière occupation du site est orientée vers la production de Dr. $2 / 4$ et de quelques amphores à fond plat. Les voies d'exportation des amphores vinaires d'Albinia vers la Gaule semblent privilégier l'axe Rhône-Saône et celui de la Loire.

15, C. Capelli, R. Cabella, M. Piazza, «Albina o no Albina? Analisi in microscopia ottica su anfore Dressel 1 rinvenute in Francia méridionale », dans F. Olmer (éd.), Itinéraires des vins romains en Gaule, Monographies d'Archéologie Méditerranéenne n5, Lattes, 2013, p. 479-483, on a souvent attribué à l'atelier d'Albina 
nombre d'amphores exportées de façon peut-être excessive. Ce premier travail d'analyse microscopique optique donne une vision plus réaliste. À partir d'un corpus d'amphores timbrées trouvées dans l'atelier, Albina se définit comme un groupe homogène qui doit recouvrir aussi des ateliers voisins encore inconnus. Des échantillons d'amphores de sites gaulois ou d'épaves de la côte gauloise dont on pouvait penser qu'ils pouvaient provenir d'Albina, ont été comparés au groupe de référence. Ainsi pour l'oppidum de l'Ermitage à Alès, sur 40 échantillons analysés, 18 viennent effectivement d'Albina. Pour Toulouse, sur 8 échantillons, 2 sont attribués à Albinia. À Agen, sur deux échantillons, l'un vient d'Albina. Pour l'épave de Miladou, un sur deux, pour celle de La Ciotat les résultats ne sont pas concluants.

16, V. Reveilla, «Las ánforas norteafricanas del Monte Testaccio (Roma): tipologías, cronologías y zonas de procedencia », dans A. Ribera i Lacomba (éd.), Manuel de cerámica romana. Del mundo Helenistico al Imperio Romano, Madrid, 2013, p. 415-441, fait le point sur les importations d'amphores de Tripolitaine, de Tunisie et de Maurétanie césarienne au Monte Testaccio.

17 , E. Botte, « Les amphores en contexte funéraire, des éléments de remploi », dans W. Van Andrigua, H. Duday, S. Lepetz, D. Joly, T. Lind (éds), Mourir à Pompéi, Fouille d'un quartier funéraire de la nécropole romaine de Porta Nocera (2003-2007), II, École française de Rome, 2013, p. 1091-1104, les amphores, assez rares, sont toutes utilisées en remploi : coffrage et couverture de sépulture, réceptacle funéraire, tube à libation et couvercle de tube à libation surtout, urne cinéraire enfin, dans ces contextes qui ne sont pas aristocratiques. La nécropole est utilisée entre les années 40-30 avant notre ère et 79, année de l'éruption du Vésuve. On y trouve en majorité des amphores italiennes: Dr. 2/4 de l'aire vésuvienne, exceptionnellement des Dr. 1 ou 21-22. Les amphores de Bétique sont rares, les Africaines plus fréquentes, les amphores crétoises sont également réutilisées.

18, G. Olcese, S. Giunta, I. Iliopoulos, C. Capelli, « Indagini archeologiche e archeometriche preliminari sulle anfore di alcuni relitti della Sicilia (metà III-I sec. a. C.) », dans F. Olmer (éd.), Itinéraires des vins romains en Gaule, Monographies d'Archéologie Méditerranéenne n5, Lattes, 2013, p. 485-512, dans le cadre du projet IMMENSAAEQUORA qui vise à reconstruire le commerce en Méditerranée à l'époque hellénistique et romaine, les auteurs présentent une étude archéologique et archéométrique des amphores gréco-italiques (essentiellement) de quatre épaves de Sicile : l'épave Terrasini B a un chargement hétérogène, celui de Capo Passero se réfere à la Sicile ou à la Grèce, celui de Filicudi A à la zone pompéienne, celui de Porto Palo di 
Menfi, comportant notamment des gréco-italiques et des Dressel 1 , se rattache au Latium et à la Toscane méridionale, mais aussi à la Campanie et peut-être à la zone adriatique.

\section{- Dans la péninsule lbérique}

19, Dans le cadre d'Ex Hispania Amphorae, ICAC, Tarragone, sous la direction de R. Járrega et P. Berni a été mis en ligne le Catalogue interactif des amphores hispaniques : http://amphorae.icac.cat/. Trente ans après l'étude de M. Beltran Lloris, La anforas romanas en España, on trouvera dans ce catalogue bilingue (espagnol, anglais), la mise à jour de la totalité des formes d'amphores produites dans la péninsule Ibérique, avec carte, typologie, bibliographie, liste alphabétique des types et liste par auteurs et, en outre, les liens avec les bases complémentaires : Céramopôle (Aix-en-Provence), Terres d'amphores (Paris-Nanterre), Roman Amphorae : a digital ressource (Southampton) et Corpus Ceipac (Barcelone). Cet outil particulièrement utile, fait partie d'un projet ouvert de laboratoire virtuel beaucoup, plus ambitieux, dont les objectifs sont les suivant :

- mise en réseau des recherches sur les amphores hispaniques,

- standardisation des typologies amphoriques,

- étude des centres de production et modélisation des études comprenant les caractérisations archéométriques,

- épigraphie amphorique,

- cartes de production et circulation,

- espace de travail et d'échange.

\section{Hispanie Citérieure et Tarraconaise}

20, J. Molina Vidal, « Commerce et marchés de vin italique dans le sud l'Hispanie Citérieure (III $-\mathrm{I}^{\mathrm{er}}$ siècle av. notre ère) », dans $\mathrm{F}$. Olmer (éd.), Itinéraires des vins romains en Gaule, Monographies d'Archéologie Méditerranéenne n5, Lattes, 2013, p. 195211, donne une analyse générale très documentée de la situation dans cette région du sud-est de l'Espagne. Les premières amphores gréco-italiques arrivent dans la seconde moitié du III ${ }^{\mathrm{e}}$ siècle avant notre ère, dans un contexte habitué aux vins grecs, puniques et punico-ébusitains. Les importations italiques de gréco-italiques sont importantes au cours du $\mathrm{II}^{\mathrm{e}}$ siècle avant notre ère, liées à la présence de l'armée et de population italienne. L'épave Escombreras I dans la baie de Carthagène en est un bon exemple, du milieu du $\mathrm{II}^{\mathrm{e}}$ siècle avant notre ère. Elle a un intérêt particulier pour la typologie car elle contient à la fois des gréco-italiques et celles dites de transition ou évoluées. Pendant l'époque tardo-républicaine, les importations de vin italien dans des Dr. 1 du Latium et de Campanie et des vins apulo-adriatiques (Lamboglia 2) sont très importantes avec des 
nuances significatives au nord et au sud du cap de la Nao : au nord, jusqu'à Ampurias, un maximum de vin tyrrhéniens, $90 \%$, pour $10 \%$ de vins apuliens, tandis qu' au sud, dans la région des mines, on trouve $50 \%$ de Dr. 1 et 50\% de Lamboglia 2. Le besoin de quantités massives d'esclaves expliquerait la relation préférentielle de cette zone avec l'Apulie dont on sait le rôle majeur dans le trafic d'esclaves orientaux à Délos. Le déclin des sources d'approvisionnement en main d'œuvre servile et le développement de l'agriculture dans les provinces sont deux facteurs déterminants pour expliquer le déclin des importations italiennes au cours du $\mathrm{I}^{\text {er }}$ siècle avant notre ère, et l'apparition d'amphores locales dès le premier tiers du $\mathrm{I}^{\mathrm{er}}$ siècle avant J.-C. qui seront ensuite remplacés par des modèles plus standardisés. Cet article fournit aussi une belle illustration épigraphique des timbres des diverses amphores et des graphiques de distribution qui, cependant, auraient peut-être mérité quelques précisions.

21, J. Tremoleda Trilla, P. Castanyer Masoliver, « Las ánforas republicanas itálicas de Catalunya (siglos III-I a. C.): estado de la cuestión », dans F. Olmer (éd.), Itinéraires des vins romains en Gaule, Monographies d'Archéologie Méditerranéenne $\mathrm{n}^{\circ} 5$, Lattes, 2013, p. 213-256, donne, à partir d'exemples précis, une vision historique de la Catalogne aux trois premiers siècles avant notre ère, dans laquelle il replace d'abord les importations importantes d'amphores gréco-italiques de Grande-Grèce au -III siècle à côté de la présence d'amphores ibériques; il souligne ensuite au $-\mathrm{II}^{\mathrm{e}}$ siècle l'ampleur de l'immigration italienne en Espagne attirée par les ressources économiques de la péninsule, dont les mines, et le développement des relations commerciales avec l'Italie. Dans ce cadre, arrivent encore des amphores gréco-italiques puis Dr. 1A, le vin italien atteint de l'ordre de 60\% à Ampurias et 70\% à Tarragone. Au -I ${ }^{\text {er }}$ siècle avec la fondation de nombreuses cités en Tarraconaise et la restructuration du territoire, on constate une domination complète du marché par les amphores vinaires Dr. 1 italiques associées à des amphores à huile de Brindes. La consommation de vin italien à tous les niveaux est alors un élément de romanisation. Durant la première moitié $\mathrm{du}-\mathrm{I}^{\mathrm{er}}$ siècle commence à se développer une viticulture propre, tandis que la politique de César s'accompagne d'une refondation de plusieurs cités et de nouveaux cadastres. C'est dans ce nouveau cadre que La Tarraconaise romanisée se met à produire massivement du vin et à l'exporter.

22, P. Berni Millet, J. Miró Canals, « Dinámica socioeconómica en la Tarrconense oriental a finales de la República y comienzos del imperio. El comercio del vino a través de la epigrafía anfórica », dans Tarraco, Actes du premier congrès international d'archéologie du monde antique, Hommage à G. Alföldy, Tarragone, 2013, p. 63-83, signent un article remarquable sur la dynamique du développement de la Tarraconaise orientale d'après 
l'apport des productions d'amphores à la fin de la République et au début de l'Empire. Les divers types produits, Dr. 1, Tarraconaise 1, Pascual 1, Oberaden 74 et Dr. 2/4, ne se succèdent pas chronologiquement pas hasard mais pour des raisons commerciales. Notamment, aux lourdes Pascual 1 de création locale, succèderont les Dr. 2/4 plus légères, adaptant un modèle qui pourra concurrencer les vins de qualité, comme ceux venus d'Orient. L'analyse des timbres liés aux formes met en lumière une évolution chronologique: praenomen et nomen pour les plus anciens (Dr. 1, Tarraconaise 1 et premières Pascual 1). Au changement d'ère, apparaissent les tria nomina sur les Pascual 1, sur lèvre ou col, auxquels succèdent au Haut-Empire, imprimés sur le pied, les cognomina (complets ou abrégés) des esclaves et des timbres anépigraphiques, mais aussi de fréquents graffites avant cuisson, toujours placés sur les pieds, marque des artisans de diverses firmes. Les Oberaden 74 sont signées par des personnages libres, duo ou tria nomina, et des noms serviles complètement développés. Les Dressel 2/4 portent aussi bien sur lèvre, sur col, sur attache inférieure de l'anse que sur le pied, des tria nomina abrégés et peu de duo nomina considérés comme archaïques, mais surtout des cognomina d'esclaves grecs ou latins.

L'adaptation du modèle Dr. 2/4 correspond à la fondation de Barcino et au remodelage de la région avec redistribution des terres. La grande industrie du vin de la région du Baix Llobregat, aux mains de l'élite coloniale romaine, s'accélère sous Tibère et ne décroîtra que sous les Flaviens. Les exportations de vin en amphores ou en dolia sont orientées vers Rome et Carthage. Il s'agit de contrecarrer l'expansion des exportations du vin de la Narbonnaise qui a quasiment fermé le marché gaulois si prospère pour le vin de Tarraconaise au moment de la grande diffusion des Pascual 1, à la fin de la République et au débout du règne d'Auguste.

23, R. Járrega Domínguez, «El port romà de Barcino (Barcelona) i el Praefectus orae maritimae. Un possible portus commercial », Bulletí arquelogic, V, 33, 2011, p. 81119, la fondation de la cité de Barcelone vers les années 10 avant J.-C. s'accompagne probablement de la création d'un port par lequel seront exportées les amphores vinaires de la zone du Llobregat et où se faisaient les formalités imposées par le portorium. À l'époque flavienne, cessent les exportations vinaires au moment où est créée la préfecture orae maritimae laeetaniae.

24, C. Carreras Monfort, A. López Mullor, J. Guitart i Duran (éds), Barcino II. Marques $i$ terrisseries d'amfores al Baix Llobregat, Corpus des timbres amphoriques fasc. 18, Institut d'Estudis Catalans, Institut Català d'Aqueologia Clàssica, Barcelone, 2013, fait suite et complète le premier volume paru en 2009, Barcino I. Les marques $i$ 
terrisseries d'àmfores al pla de Barcelona, en soulignant le dynamisme de la recherche sur les amphores dans cette région. Une vision globale de la production du Baix Llobregat se dessine : 25, C. Carreras Monfort, A. López Mullor, J. Guitart i Duran, «El Baix Llobregat: una vall vinícola i terrissera de l'ager barcionensis », p. 11-16, dans la partie centuriée de la colonie de Barcino, la production viticole s'intensifie après la période républicaine : en témoignent l'atelier de San Boi de Llobregat avec des Dr. 2/4 à partir de l'époque de Tibère, ceux de Sant Vicenç dels Horts, de Can Pedrerol de Baix et de Can Tintorer, par exemple. Les exportations se dirigent dans un premier temps vers le Golfe du Lion, l'axe Aude-Garonne et le limes germanique, en particulier à Neuss et Nimègue, puis avec la croissance qui correspond à l'époque de Tibère, vers Rome, où 70\% des Dr. 2/4 de Tarraconaise du port du Monte Testaccio viennent du Baix Llobregat. 26, L. Moret Pujol, C. Carreras Monfort, C. Miró i Alaix, « Terrisseries al volant de la riera de Rubí: Can Tintorer (El Papiol) i Can Pedrerol de Baix (Castellbibal) », p. 17-59, ces deux officines liées à la production du vin fabriquent des Pascual 1 mais surtout des Dr. 2/4 que l'on transportera facilement par la voie fluviale. Le point est fait sur le déroulement des recherches qui y ont été conduites depuis de nombreuses années. 27, J. Miquel i López, J. Morera Campbrubí, « San Vicenç desl Horts: una zona productora de tipus industrial en època altimperial romana $\gg$, p. 33-50, des fouilles récentes ont mis au jour une partie de la zone de production. L'officine s'est développée entre 10 avant notre ère et la fin du premier siècle de notre ère-début du $\mathrm{II}^{\mathrm{e}}$ siècle. La production sera majoritairement des Pascual 1 dans un premier temps, puis des Dr. 2/4. On compte dix-huit timbres différents dont la marque SYN-SYNE en abondance. 28, M.-L. Barreda i Casanova, « La terrisseria del jaciment del Barri Antic (Sant Boi de Llobregat) », p. 51-59, fait l'historique des recherches jusqu'aux plus récentes, et propose une évolution du site, fours en activité et timbres. 29, A. López Mullor, « La figlina i les àmfores de l'àrea de les termes romanes de San Boi de Llobregat », p. 61-81, fait le point sur l'atelier et ses productions de Pascual 1 et Dr. 2/4. 30, J. M. Huélamo Gabaldón, J. M. Solias i Arís, « Can Manyoses (Viladecans, Baix Llobregat): l'abrocador d'un nou centre productor de ceràmica », p. 83-96, l'atelier produit des Tarraconaises 1, Pascual 1 et Dr.2/4, le seul timbre connu est PRI. 31, V. Martínez Ferreras, A. Gutiérrez García-Moreno, « Caracterizació arqueomètrica de les àmfores produïdes a les terrisseries del Baix Llobregat », p. 97-126, présentent un travail très important et complet d'analyses chimiques, minéralogiques et pétrographiques des centres producteurs de la Via Vella, Can Reverter, Can Tintorer et Can Pedrerol. Il apparaît que les processus technologiques sont standardisés. La région du Baix Llobregat est dorénavant bien caractérisée d'un atelier à l'autre. On note aussi des différences dans les productions de Pascual 1 et Dr. 2/4. 32, P. Berni Millet, 
C. Carreras Monfort, « Corpus epigràfic de segells en àmfores, dolia, tegulae i gerres de ceràmica comuna oxidada del Baix Llobregat », p. 127-285, il était intéressant de prendre en compte ces diverses céramiques pour montrer la prospérité économique de la région des dernières années avant notre ère au milieu du $\mathrm{I}^{\mathrm{er}}$ siècle. La majorité des timbres sur Dr. 2/4 datés de l'époque de Tibère, montre à quel point le développement économique de la région et l'exportation du vin étaient forts à cette période. Le corpus compte 122 timbres différents (lisibles) sur amphores locales, ils sont attribués à des ateliers précis. Suivent quelques timbres sur amphores d'importation. Faisant suite aux travaux pionniers de R. Pascual Guash, ce nouveau corpus mis à jour et largement complété, est un outil remarquable pour les chercheurs. 33, D. Gorostidi Pi, « Sobre les marques SYN/SYNE i la seva identificació amb C. TROCINA SYNECDEMUS, sevir augustal de la colónia de Barcino », p. 287-296, ou la carrière fulgurante d'un personnage : esclave dans sa jeunesse, il timbre des amphores à Sant Vicenç dels Horts, puis devient un affranchi de la célèbre famille Trocina de Barcino et plus tard sevir augustal de la cité ! 34, P. de Soto Cañamares, « Anàlisi de la distribució il la mobilitat en el terrotori del riu Rubricatum », p. 297-308, montre comment la création d'un réseau de voies liées à la fondation de Barcino, comme la présence du Llobregat vont favoriser le développement agricole et économique de la région. 35, C. Carreras Monfort, « Evolució de les terrisseries del Baix Llobregat a partir de les seves marques i els seus derelictes », p. 323-346, fait une étude croisée des ateliers du Baix Llobregat, de la chronologie de leurs timbres et du chargement d'une série d'épaves. En ressortent une série de résultats, notons en particulier que les commerçants n'achetaient pas les amphores pleines issues d'un seul atelier mais de toute une région de production.

\section{Bétique}

36, J. Remesal, « Nuevos datos sobre las confiscaciones de Septimo Severo en la Bética, » dans Tarraco, Actes du premier congrès international d'archéologie du monde antique, Hommage à G. Alfoldy, Tarragone 2013, p. 233-245, les nouvelles découvertes épigraphiques sur Dr. 20 au Monte Testaccio à Rome montrent que certains timbres du III ${ }^{\mathrm{e}}$ siècle, des figlinae Barba, Ceparia et Grumensis sont concentrés dans les mêmes niveaux datés de 204 et 205 de notre ère. Ainsi apparaît-il que la crise du III ${ }^{\mathrm{e}}$ siècle ne peut être traitée de façon générale, mais qu'il convient d'analyser la situation particulière de chaque province de l'Empire.

37, P. Berni Millet, D. Gorostidi Pi, « C. Iulianus Valerianus et C. Iulius Iulianus: mercatores del aceite bético en un signaculum de plomo para ánforas Dressel 20 », Journal of Roman Archaeology, 26, 2013, p. 167-189, la découverte rare d'un signaculum 
de plomb, est l'occasion de faire un état de la question sur les commerçants d'huile de Bétique de la gens Iulia, famille bien connue sur les inscriptions $\beta$ des Dr. 20. On s'interroge par ailleurs sur le mode d'obturation des amphores à huile, disque d'argile et couche de mortier de chaux qui pouvait être imprimée par un signaculum dont l'inscription est un contrôle supplémentaire à valeur commerciale.

38, E. García Vargas, « Hispalis (Sevilla, España) y el comercio mediterráneo en el alto Imperio romano. El testimonio de las ánforas », dans S. Keay (éd.), Portus and the Mediterranean, Archaeology Monographs of the British School at Rome, 21, 2012, p. 245-266, présente et analyse le matériel amphorique des fouilles récentes et plus anciennes de l'aire portuaire de Séville. On y compte notamment plusieurs importations gauloises. Séville était aussi un centre de productions d'amphores : Dr. 20 en majorité, Dr. 28, H. 70 et Beltran IIA. Le port d'Hispalis a été actif pendant toute la période romaine et jusqu' aux $\mathrm{V}^{\mathrm{e}}$ et $\mathrm{VI}^{\mathrm{e}}$ siècle. Son activité maximale se situe au $\mathrm{I}^{\text {er }}$ siècle et jusqu'aux premières années du $\mathrm{II}^{\mathrm{e}}$ siècle. Dans le domaine des importations, on souligne la connexion intense avec le port de Narbonne d'où vient la quasi-totalité du vin importé au I ${ }^{\text {er }}$ siècle. La quasi absence des vins de Tarraconaise montre qu' il y avait une relation directe entre Narbonne et Séville. Un panorama des exportations d'huile depuis Séville est dressé.

39, E. García Vargas, D. Bernal Casasola et al., « Confectio gari pompeiani. Procedimiento experimental para elaboración de salsas de pescado romanas », SPAL, Revista de Prehistoria y Arqueología, 23, 2014, p. 65-82, en coopération les universités de Cadix et de Séville, avec la participation d'archéologues et de biochimistes, ont tenté de fabriquer les diverses sauces de poisson romaines : garum (semblable au liquamen), hallec, et muria en partant des renseignements fournis par les textes anciens et des analyses de résidus antiques. L'expérience est un succès.

\section{- Dans les Gaules}

40, A. Tchernia, « Transport et choix des vins : quelques règles », dans F. Olmer (éd.), Itinéraires des vins romains en Gaule, Monographies d'Archéologie Méditerranéenne $\mathrm{n}^{\circ} 5$, Lattes, 2013, p. 531-534, on souscrira bien volontiers aux deux préceptes proposés par A. Tchernia : 1, le vin transporté par terre avant l'arrivée du chemin du fer est cher et son prix à l'arrivée n'a plus rien à voir avec son prix au départ. 2 , il y discordance entre les goûts des consommateurs italiens et gaulois. Les premiers, on le sait, apprécient le plus les vins blancs vieillis. Les second sont attirés depuis le VI siècle au moins par le 
rouge, couleur du sang, le corail d'abord, puis le vin qu' ils boivent pur en suivant des rites sacrificiels. Des goûts et des couleurs... et des façons de consommer pourrait-on ajouter !

41, Depuis décembre 2013 est mise en ligne sur le site de la Maison de l'Archéologie de Nanterre, dans le cadre de l'UMR 7041, Équipe GAMA, sous la direction de F. Laubenheimer et E. Marlière, la base de données Terres d'amphores: http://www.mae.u-paris10.fr/terresdamphores/. Elle concerne les échantillons de pâtes d'amphores des ateliers de l'ensemble de la Gaule. Chaque atelier est représenté par plusieurs échantillons avec pour chacun une fiche descriptive, une macro-photo de la section et de la surface de la pâte (plus d'un millier de photos), une vignette de la forme de l'amphore, et la bibliographie de l'atelier. On trouvera aussi un lien avec la base de données analytique (fluorescence $\mathrm{X}$ ) des ateliers d'amphores vinaires de Narbonnaise (MOM, Lyon).

42, B. Dedet, J. Salles, «L'Ermitage d'Alès (Gard): un oppidum-marché du $\mathrm{I}^{\mathrm{er}}$ siècle avant J.-C. et la question des antécédents de la voie cévenole », dans F. Olmer (éd.), Itinéraires des vins romains en Gaule, Monographies d'Archéologie Méditerranéenne n ${ }^{\circ} 5$, Lattes, 2013, p. 23-38, donnent une nouvelle présentation de cette place commerciale du I ${ }^{\mathrm{er}}$ siècle avant J.-C. Créée ex nibilo au début du $\mathrm{I}^{\mathrm{er}}$ siècle avant notre ère, elle a cessé d'exister dans les années 30 avant notre ère. Implanté à la porte des Cévennes et du Massif central, le site présente des indicateurs économiques d'échanges avec la Gaule centrale plus abondants qu'ailleurs dans la région : quantité d'amphores italiques, nombreuses monnaies de la Gallia Comata, principalement du centre de la Gaule, un élevage porcin insolite en Languedoc, des bols hellénistiques et de la céramique celtique tournée. Les maisons sont plus vastes que dans le voisinage et couvertes de tuiles. On compte aussi d'autres marqueurs d'influence romaine précoce, comme l'écriture ou la consommation de coquillages loin de la mer. L'Ermitage a visiblement été créé comme relais des importations de vin italien vers le centre de la Gaule sur une voie transcévenole qui n'existait pas auparavant entre la province de Gaule transalpine et la Gaule indépendante. L'Ermitage est un cas remarquable par sa liaison directe au commerce, il n'a vécu que le temps des importations massives de vins italiens.

43, E. Gomez, Les productions de vin et d'amphores tardo-hellénistiques à Saint-Michel (Agde-34), dans F. Olmer (éd.), Itinéraires des vins romains en Gaule, Monographies d'Archéologie Méditerranéenne n5, Lattes, 2013, p. 39-56, nombre d'exploitations agricoles de la deuxième moitié du $\mathrm{II}^{\mathrm{e}}$ siècle av. J.-C. ont été découvertes dans l'ancienne chôra d'Agde, dont plus d'une douzaine sont liées à la production de vin. Saint-Michel en fait partie, inscrit dans un territoire cadastré. Il présente des installations 
viticoles et un atelier de potiers fabriquant des tuiles, peut-être des dolia et surtout des amphores de type italique caractéristiques de la deuxième moitié du $\mathrm{II}^{\mathrm{e}}$ siècle av. notre ère. L'auteur nous apporte un regard nouveau sur la production du vin en Narbonnaise précédent le grand boom de celle du Haut-Empire.

44, S. Mauné, « La production d'amphores vinaires en Gaule transalpine. État des lieux et perspectives ( $\mathrm{II}^{\mathrm{e}}-\mathrm{I}^{\mathrm{er}}$ siècle av. J.-C.) », dans F. Olmer (éd.), Itinéraires des vins romains en Gaule, Monographies d'Archéologie Méditerranéenne n5, Lattes, 2013, p. 57-73, après avoir fait un point sur la viticulture protohistorique indigène et le rôle de Marseille, S. M. rassemble les éléments qui pourraient éclairer le développement de la production de vin à la fin du $\mathrm{II}^{\mathrm{e}}$ siècle et au I ${ }^{\text {er }}$ siècle avant notre ère. Quelques ateliers produisant des Dr. 1 en Languedoc occidental et dans la vallée du Rhône sont bien identifiés, d'autres sont pressentis et demandent des compléments de recherche ; les Dr. 1 dont les timbres très particuliers comportent le mot MESOR (mensor, contremaître), distribuées essentiellement dans l'axe Aude-Garonne pourraient bien être d'origine locale, tout comme certaines amphores Dr. 1 à pâte orangée. Un parallèle est établi avec les officines de Dr. 1 de Catalogne qui traduisent une viticulture coloniale active dès la première moitié du $\mathrm{I}^{\mathrm{er}}$ siècle avant notre ère. Autant de pistes de recherches dynamiques et cohérentes qui méritent d'être suivies pour mieux appréhender le grand développement de la viticulture Narbonnaise impériale.

45, S. Barberan et al., « Le vin dans les pratiques funéraires du Midi de la Gaule aux II ${ }^{\mathrm{e}}-\mathrm{I}^{\text {er }}$ siècle av. J.-C. : l'exemple du Mas Vigier à Nîmes (Gard) », dans F. Olmer (éd.), Itinéraires des vins romains en Gaule, Monographies d'Archéologie Méditerranéenne $\mathrm{n}^{\circ} 5$, Lattes, 2013, p. 535-563, cet article très bien documenté met en valeur la tombe du Mas Vigier, isolée, en bordure de voie, comme souvent dès le - $\mathrm{II}^{\mathrm{e}}$ siècle dans la région. À l'intérieur d'un coffrage rigide, les objets ont été déposés sur un support, autour d'une amphore complète Dr. 1B d'origine pompéienne, installée en premier, en place centrale. Sept vases à boire, dont un ossuaire, des armes pliées ou brisées et des offrandes

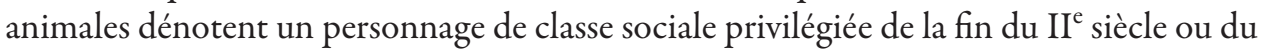
début du $\mathrm{I}^{\text {er }}$ siècle avant notre ère. Le banquet funéraire et la consommation de vin sont emblématiques des tombes nîmoises de la fin du $\mathrm{II}^{\mathrm{e}}$ siècle. L'absence de vases et objets métalliques liés au symposium, tels qu'on les rencontre dans la basse vallée du Rhône ou dans les Alpilles, caractérise une société aux pratiques indigènes plus traditionnelles.

46, A. Tchesnakoff, «Un faciès de consommation à Marseille à l'époque tardohellénistique. La céramique et les amphores d'un ensemble stratifié du site de la rue J. F. Leca (II ${ }^{\mathrm{e}}-\mathrm{I}^{\mathrm{er}}$ siècle av. J.-C. ) », dans SFECAG, Actes du Congrès d'Amiens 2013, 
p. 351-363, ce faciès souligne la diminution des amphores massaliètes et la l'importance des amphores vinaires italiques à cette période. Parmi les autres importations en quantité mineure, à souligner des amphores puniques et des amphores égéennes dont une

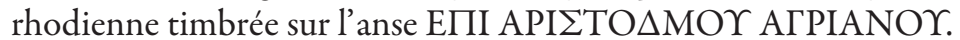

47, P. Goher, C. Capelli, «Les amphorettes levantines du dépotoir du site « 5 place Jean-Baptiste Massillon » à Arles. Un modèle miniature des amphores Célestins 1A ? Approche typologique et pétrographique », dans SFECAG, Actes du Congrès d'Amiens 2013, p. 549-554, dans un dépotoir du III ${ }^{\mathrm{e}}$ siècle cinq petites amphores d'environ $24 \mathrm{~cm}$ de haut représentent un type peu connu et pourtant bien attesté en Gaule Narbonnaise, notamment dans les ports (Marseille, Toulon). Leurs similitudes avec les amphores orientales Célestin 1A de quelque $65 \mathrm{~cm}$ de haut font penser qu'il s'agit d'un petit module du même type ce que confirment les analyses de pâte. Leur origine ne serait pas Beyrouth mais plus largement levantine ou libanaise. Leur contenu pourrait être des dattes et des olives comme celles retrouvées carbonisées dans deux Célestin $1 \mathrm{~A}$ et comme les fruits contenus dans les amphores carottes. Leur distribution semble démontrer un usage réservé à l'exportation.

48, P. Chapon et al., « Un établissement rural du Haut-Empire à vocation viticole au Clos Sainte-Anne à Rougiers (Var) », Revue Archéologique de Narbonnaise, 43, 2010, p. 155-192, présentent une villa de taille moyenne comportant des pressoirs et un chai d'une soixantaine de dolia qui permettent de stocker de l'ordre de 280 à $300 \mathrm{hl}$ de vin. L'activité viticole cesse à la fin du $\mathrm{II}^{\mathrm{e}}$ siècle, victime sans doute du développement de grands domaines voisins.

49, M. Ott, « L'exploitation agricole du mas de Fourques aux I ${ }^{\mathrm{er}}$ et $\mathrm{II}^{\mathrm{e}}$ siècle de notre ère (Lunel, Hérault) », Revue Archéologique de Narbonnaise, 43, 2010, p. 193-206, un établissement agricole modeste à vocation polyculturale a produit du vin comme en témoignent un chai de 26 dolia.

50, E. Henry, C. Raynaud, « La ferme gallo-romaine de Las Olivetas à Mudaison (Hérault) », Revue Archéologique de Narbonnaise, 43, 2010, p. 207-242, cette modeste ferme a, au I ${ }^{\text {er }}$ siècle, pratiqué un peu de viticulture et fait un peu de vin comme en témoignent pressoir et dolia.

51, B. Helly, T. Sylvino, « Un nouveau témoignage du commerce antique aux portes des Alpes: la fouille du musée d'Aoste », Revue Archéologique de Narbonnaise, 44, 2011, p. 179-204, la découverte récente d'une fosse contenant quarante amphores est rapprochée de celle d'une grande galerie contenant deux niveaux d'amphores et découverte en 1875, probablement un entrepôt lié à un macellum. Ces deux éléments 
permettent de souligner l'importance commerciale d'Aoste, zone charnière entre le Sud et les Alpes. La fosse dont le dépôt est daté des années 50/70, contient 18 amphores d'origine lyonnaise : la majorité sont des amphores à saumure, Lyon 3 surtout et Dr. 16, il y a aussi des vinaires, Dr. 2/4, Dr. 28 et H. 70, et, plus inédit, une amphore à huile Dr. 20 gauloise. Des importations de Bétique sont représentées par 13 Dr. 8 ou 11 et 6 Dr. 20. On compte également des amphores vinaires orientales : 3 Dr. 2/4 et deux Rhodiennes et, curieusement une seule amphore G. 4 de Narbonnaise.

52, C. Caratto, « Le four 3 de l'atelier de potier de Saint-Bézard et ses productions (Aspiran, Hérault). Contribution à la connaissance de l'artisanat potier en Gaule Narbonnaise », Revue Archéologique de Narbonnaise, 45, 2012, p. 39-73, de nouvelles recherches portant sur un des premiers fours augustéen de l'atelier d'Aspiran conduisent à une étude très détaillée du four 3 à sole de plan rectangulaire avec un laboratoire de 56 à $42 \mathrm{~m}^{3}$ suivant les périodes. Il a produit des amphores Pascual 1, G. 7 et G. 9 et des tuiles. Une intéressante comparaison est menée avec les fours de Narbonnaise de même époque.

53, S. Mauné, G. Duperron, «Un lot de céramiques des années 10 apr. J.-C. mis au jour à Saint-Bézard (Aspiran, Hérault) », Revue Archéologique de Narbonnaise, 45, 2012, p. 111-142, le comblement d'un fossé particulièrement riche en céramiques italiennes est bien daté. On peut attribuer aux années dix après J.-C. les quelques amphores présentes (19 NMI): Dr. 20a, Dr. 12, H.70 et Dr.7/11 de Bétique, plus original, une Dr. 14 de Lusitanie, des P. 1 de Tarraconaise dont le timbre Iuli Theophili est ici bien daté, une Dr. 2/4 de Campanie et des amphores régionales : Pascual 1 timbrée Laeti et déjà Dr. 2/4, G. 7 et G. 9.

54, S. Mauné, « Un timbre de Q. Iulius Theophilus sur Pascual 1 de Tarraconaise à Aspiran (Hérault). Un nouvel indice de liens économiques entre les provinces de Tarraconaise et de Narbonnaise au début du I ${ }^{\mathrm{er}}$ siècle? », Revue Archéologique de Narbonnaise, 45, 2012, p. 143-161, ce fameux Iulius Theophilus que nous venons d'évoquer serait-il lié à Q. Iulius Primus ou Priscus d'Aspiran ? Des indices de la présence de ce dernier sont présents dans la basse vallée du Llobregat et à Barcino. Par ailleurs, le Laetus d'Aspiran a également travaillé à Barcino à la même période. On s'interroge sur les possibilités qu'avait Q. Iulius Priscus d'intervenir dans la production du vin en Tarraconaise.

55, S. Barberan et al., «Boire et manger sur le forum du Castellas (Murviellès-Montpellier, Hérault): un dépotoir atypique de la première moitié du $\mathrm{II}^{\mathrm{e}}$ siècle apr. J.-C. », Revue Archéologique de Narbonnaise, 45, 2012, p. 293-360, il apparaît clairement que l'on consomme avant tout du vin de Narbonnaise transporté dans des 
amphores G. 1, à pâte calcaire, vouées surtout au commerce de proximité (et non des G. 4 destinées au grand commerce). À noter un timbre inédit sur G. 1 : LLS ou LES. Huile et sauces de poisson de Bétique et vin oriental sont également présents.

56, M. Passelac, «Sur la voie d'Aquitaine : les amphores d'époque républicaine d'Eburomagus », dans F. Olmer (éd.), Itinéraires des vins romains en Gaule, Monographies d'Archéologie Méditerranéenne n5, Lattes, 2013, p. 75-124, entre Carcassonne et le seuil de Naurouze, Bram est situé dans une zone de carrefour. L'analyse porte à la fois sur Bram et sur les sites ruraux environnants qui ont été largement importateurs d'amphores italiques. On constate une consommation massive de vins italiens à Bram à partir du second quart du $\mathrm{II}^{\mathrm{e}}$ siècle et surtout durant la seconde moitié du $\mathrm{II}^{\mathrm{e}}$ siècle avant notre ère, soit avant les taxations sur le vin italien rapportées par Cicéron lors du procès de Fontéius. En revanche, à l'époque du procès, vers -75 , un infléchissement notoire du trafic des Dr. 1A se fait sentir, lié sans doute aux taxations appliquées à Bram. Le site aurait-il été contourné pour cette raison ? Les Dr. 1B sont rares, elles apparaissent vers le milieu du $-\mathrm{I}^{\mathrm{er}}$ siècle et sont présentes jusqu'à la période d'Auguste. D’après les pâtes des amphores, les importations du - $\mathrm{II}^{\mathrm{e}}$ siècle proviennent de l'Italie centro-méridionale et méridionale. Comme à Toulouse, on note peu d'importations d'Étrurie méridionale : de Cosa et d'Albina. Soulignons l'illustration très riche des formes et des timbres. Pour ces derniers un catalogue documenté aurait été bien venu.

57, F. Verdin, F. Berthault, C. Sanchez, « Le puits 41 de l'oppidum de l'Ermitage d'Agen (Lot-et-Garonne) : aperçu du faciès amphorique et questions d'archéologie », dans F. Olmer (éd.), Itinéraires des vins romains en Gaule, Monographies d'Archéologie Méditerranéenne n5, Lattes, 2013, p. 125-138, ce puits a livré un matériel remarquable que l'on évalue à 208 amphores Dr. 1 et une amphore ovoïde de l'Adriatique, accompagnées de vaisselle d'importation. L'analyse typo-chronologique des amphores est finement menée en mettant en relation les variantes des formes et la chronologie apportée par neuf marques peintes comportant des dates consulaires, en regard avec la céramique campanienne $\mathrm{A}$ et $\mathrm{B}$. Il apparaît que ce relais, le plus important du commerce italique sur l'axe de la Garonne, est caractéristique de la fin du II ${ }^{\mathrm{e}}$ siècle et du premier tiers $\mathrm{du} \mathrm{I}^{\mathrm{er}}$ siècle avant notre ère.

58, L. Benquet, « Les importations d'amphores au I ${ }^{\mathrm{er}}$ siècle avant J.-C. : le faciès toulousain », dans F. Olmer (éd.), Itinéraires des vins romains en Gaule, Monographies d'Archéologie Méditerranéenne $n^{\circ} 5$, Lattes, 2013, p. 139-158, cet article fait suite à celui du même auteur en 2007 sur la situation des importations au $\mathrm{II}^{\mathrm{e}}$ siècle av. notre ère. L. B. retient quatre sites caractéristiques de la première moitié du I ${ }^{\mathrm{er}}$ siècle avant J.-C. et 
deux de la seconde moitié du siècle. Dans la première période, prédominent les Dr. 1A comme à Narbonne, Lattes ou La Lagaste, mais à la différence de Lyon, Roanne, Chartres et l'Auvergne. À côté des vins italiens arrivent déjà des sauces de poisson (Dr. 1C), de l'huile de Brindes et de Tripolitaine. La faible présence des Dr. 1B dans le Toulousain est compensée par la présence d'un type hybride, mal défini, Dr. 1A/B. Le timbre AELI+ nom servile sur ce type et sur Dr. 1B, au deuxième tiers du $\mathrm{I}^{\mathrm{er}}$ siècle av. J.-C., serait caractéristique d'une distribution vouée aux marchés de l'isthme gaulois. Dans la deuxième moitié du $\mathrm{I}^{\mathrm{er}}$ siècle, caractérisée par l'arrivée d'un fort contingent d'amphores espagnoles, la présence d'amphores Dr. 1 reste bien affirmée jusqu'à la période augustéenne. Il en ressort une nette différenciation entre les importations de l'axe rhodanien et celles de l'axe audois.

59, F. Berthault, « Bordeaux et le faciès girondin : entre Dressel 1 et Pascual 1 », dans F. Olmer (éd.), Itinéraires des vins romains en Gaule, Monographies d'Archéologie Méditerranéenne n5, Lattes, 2013, p. 159-177, note l'absence d'amphores italiques dans les basses vallées de la Dordogne et de la Garonne avant le milieu du $\mathrm{II}^{\mathrm{e}}$ siècle avant notre ère. Leur arrivée en masse se place à la fin du siècle et au début du suivant. Les provenances sont la Campanie, le Latium et l'Étrurie. Comme on le savait, les vins de Tarraconaise succèderont aux vins italiens par la suite, avec un maximum d'importations au moment du changement d'ère.

60, J.-M. Séguier, L. Izac-Imbert, «Les amphores italiques de deux sites du sud-Albigeois : Castres, «Lameilhé » et Montfa, «La Chicane » (Tarn)», dans F. Olmer (éd.), Itinéraires des vins romains en Gaule, Monographies d'Archéologie Méditerranéenne n 5 , Lattes, 2013, p. 179-194, à la charnière de la Gaule Narbonnaise et de la Gaule indépendante se trouve le puits de Lameilhé comblé d'amphores Dr. 1 entières ou sabrées ( 32 exemplaires) et d'autre matériel lié au vin. Ce puits rituel de la fin $\mathrm{du} \mathrm{II}{ }^{\mathrm{e}}$ siècle ou du début du I ${ }^{\mathrm{er}}$ siècle avant notre ère, $s^{\prime}$ inscrit dans la tradition rituelle gauloise de consommation du vin italien, au reste bien développée dans la région. Les fosses et fossés de La Chicane riches en amphores italiques sont associés à un contexte d'habitat et d'exploitation minière et ne constituent pas un ensemble homogène que l'on peut situer au dernier tiers du II $^{\mathrm{e}}$ siècle.

61, C. Barthelémy-Sylvand, «Les amphores républicaines en Région Centre: hiérarchie des voies commerciales », dans F. Olmer (éd.), Itinéraires des vins romains en Gaule, Monographies d'Archéologie Méditerranéenne n5, Lattes, 2013, p. 275-285, plus de 2000 lèvres d'amphores gréco-italiques et Dr. 1 de chronologie incertaine, sont classées typologiquement et ainsi ordonnées dans le temps suivant le rapport HL/EL, méthode quelque peu téméraire. Il apparaît que les importations vinaires italiques sont 
beaucoup plus nombreuses chez les Bituriges que chez leurs voisins les Carnutes et les Turons. Deux voies d'approvisionnement se dessinent, la voie majoritaire par la vallée de la Loire et la route du sud du territoire des Bituriges.

62, V. Martínez Ferreras, «La diffusion commerciale des amphores vinaires de Tarraconaise à Lattara (Lattes, Hérault) », dans F. Olmer (éd.), Itinéraires des vins romains en Gaule, Monographies d'Archéologie Méditerranéenne n5, Lattes, 2013, p. 257-273, trente-et-une amphores de Tarraconaise trouvées dans des contextes datés de Lattes ont été analysées par fluorescence X et comparées à la base de données composées de 402 amphores issues de divers ateliers des territoires de Tarraco, de Barcino, de Baetulo et d'Illuro. Les premiers vins arrivent à Lattes entre le deuxième et le troisième quart du $\mathrm{I}^{\text {er }}$ siècle av. J.-C. dans des amphores de Cabrera de la Mar (Illuro) à pâte rougeâtre. Au dernier quart du $-\mathrm{I}^{\mathrm{er}}$ siècle, ce sont des amphores à pâte marron des ateliers du Barric Antic, de Can Peixau et de Calonge, du territoire de Baetulo et de la basse vallée du Llobregat. Au I ${ }^{\text {er }}$ siècle de notre ère, arrivent des productions de la colonie de Barcino (Ca l'Arnau et rue Princesa à Barcelone) à pâte claire et orangée. Tous les échantillons ne se sont pas vus attribuer une origine, l'étude d'autres ateliers est en cours pour élargir la base de référence. Mais déjà, pour la première fois en Gaule, est présentée une mise en perspective des importations de Tarraconaise avec des variations en fonction de leur origine et de leur époque.

63, Y. Barat, F. Laubenheimer, «Importation du vin chez les Carnutes de la Tène finale à Auguste », dans F. Olmer (éd.), Itinéraires des vins romains en Gaule, Monographies d'Archéologie Méditerranéenne n5, Lattes, 2013, p. 287-294, deux agglomérations, l'Île-Belle - les Mureaux et Jouars Ponchartin ainsi que deux habitats ruraux, Palaiseau et Richebourg, sont présentés en exemple d'une étude qui recouvre plusieurs centaines de sites de la partie orientale de l'Île-de-France. La présence de vin italique est banale mais pas automatique, notamment dans les sanctuaires. Des amphores gréco-italiques tardives sont arrivées dès la fin du -II ${ }^{\mathrm{e}}$ siècle. La majorité des amphores correspond à des Dr. 1, les Dr. 1B sont rares mais présentes. À Palaiseau, les importations d'Étrurie sont conséquentes (31\%) dès la période 150-80 avant notre ère. À l'époque augustéenne, on compte peu de vins de Tarraconaise, quelques vins d'Orient dans la riche villa de Richebourg, et un peu de vin de Marseille.

64, J.-M. Séguier, « La consommation des vins italiens chez les Sénons, les Meldes et les Parisii », dans F. Olmer (éd.), Itinéraires des vins romains en Gaule, Monographies d'Archéologie Méditerranéenne n5, Lattes, 2013, p. 295-314, dans la partie orientale et centrale de l'Île-de-France, les amphores gréco-italiques apparaissent dès la première moitié du II siècle av. J.-C. Puis, dans la seconde moitié du -II ${ }^{\mathrm{e}}$ et la première du - $\mathrm{I}^{\text {er }}$ siècle 
les vins tyrrhéniens sont abondants comme le montrent plusieurs exemples quantifiés et datés. Les sites ruraux sont aussi concernés, mais l'abondance de vin est plus importante sur ceux à caractère aristocratique. Les contextes parisiens suggèrent que le commerce du vin italien est encore très actif après la conquête et jusqu’à la période augustéenne, avec des Dr. 1 à lèvre courte et des Dr. 1B. Des exemples remarquables soulignent le rôle primordial du vin dans les activités rituelles et collectives liées à la sphère aristocratique. Un catalogue de timbres sur Dr. 1 complète l'article.

65, F. Laubenheimer, Y. Menez, S. Le Forestier, « Les amphores de Paule (Côtes d'Armor) et le commerce du vin au second âge du fer dans le nord-ouest de la Gaule », dans F. Olmer (éd.), Itinéraires des vins romains en Gaule, Monographies d'Archéologie Méditerranéenne n5, Lattes, 2013, p. 315-325, le site princier de Paule a fourni à lui seul plus d'amphores vinaires italiques que la totalité des sites contemporains de la péninsule armoricaine (7 449 tessons) ici cartographiés. On compte quelques amphores gréco-italiques au début du $\mathrm{II}^{\mathrm{e}}$ siècle avant notre ère. La majorité des lèvres d'amphores italiques des $\mathrm{II}^{\mathrm{e}}$ et $\mathrm{I}^{\mathrm{er}}$ siècle avant $\mathrm{J}$.-C. sont courtes et ne montrent aucune évolution typologique dans le temps. Il n'y a qu'une amphore Dr. 1B. De schémas comparables, étudiés de la même façon, se présentent sur l'oppidum de Châteaumeillant et dans les fermes du Chemin Chevaleret (Deux-Sèvres) et des Genâts (Vendée). L'observation des pâtes montre à Paule l'arrivée de quelques amphores d'Étrurie dès le début du -II ${ }^{\mathrm{e}}$ siècle.

66, G. Videau, « Les amphores tardo-républicaines dans les habitats ruraux de la vallée de la Saône et la vallée du Doubs », dans F. Olmer (éd.), Itinéraires des vins romains en Gaule, Monographies d'Archéologie Méditerranéenne n5, Lattes, 2013, p. 345-369, trois sites sont pris en compte dans le détail, d'autres sont utilisés à titre comparatif. On observe une différence pour les lèvres de Dr. 1 entre les faciès LTD1, lèvres courtes triangulaires et LTD2, lèvres plus longues en bandeau. Il s'agit en réalité de tendances qui demandent à être confirmées par les caractéristiques d'un matériel plus complet. Des pâtes du Latium, de Campanie (LTD1) et d'Étrurie (LTD2) sont repérées.

67, C. Chaidron, S. Dubois, « DeLa Tène finaleàl'époque augustéenne : premiers rapports du monde méditerranéen dans le nord-est de la Gaule », dans SFECAG, Actes du Congrès d'Amiens 2013, p. 13-64, une enquête très précise sur la distribution des amphores vient utilement compléter la synthèse parue en 2010 sur le nord-ouest de la Gaule (F. Laubenheimer, E. Marlière), notamment avec les travaux du canal Seine-Nord Europe. Il apparaît, pour les populations les plus septentrionales, que la distribution des Dr. 1 concerne l'ensemble du territoire, pour de petites quantités. Le milieu rural (une ferme sur deux) est concerné, comme les sites de pouvoir ou les sanctuaires. Si la majorité 
des amphores vinaires italiques arrivent au milieu du $\mathrm{I}^{\text {er }}$ siècle avant notre ère, d'autres sont postérieures à la conquête et arrivent encore largement après puisqu' elles figurent sur des sites augustéens créés ex nibilo. Il se confirme par ailleurs que les importations de vin de Tarraconaise sont très rares et concernent de préférence les chefs-lieux de cité, voire, en milieu rural, des établissements à caractère aristocratiques.

68, C. Chaidron, G. Fercoq du Lesay, «Éléments céramiques précoces dans les contextes cultuels du sanctuaire gallo-romain de Ribemont-sur-Ancre (Somme) », dans SFECAG, Actes du Congrès d'Amiens 2013, p. 221-234, donne une vision actualisée des amphores italiques républicaines et impériales. À signaler deux nouveaux timbres : M. Aem(ilius) Rus(ticus) sur Dr. 20, déjà connu à Vandeuil Caply, et (Mat)uri sur anse Gauloise 4.

69, L. Long, G. Volpe, M. Turchiano, « L'épave tardo-républicaine de La Ciotat : les amphores, la céramique et les hypothèses épigraphiques », dans F. Olmer (éd.), Itinéraires des vins romains en Gaule, Monographies d'Archéologie Méditerranéenne n 5 , Lattes, 2013, p. 453-483, la fouille partielle en 2001 de l'épave de La Ciotat par $57 \mathrm{~m}$ de fond a livré 278 amphores de type Dr. 1A, massif, proche des Dr. 1B. Plusieurs variantes ont été reconnues. L'épave comportait aussi un chargement de céramique commune. 110 amphores étaient timbrées correspondant à 14 timbres différents et 11 difficiles à lire. La majorité des amphores est timbrée sur le coude de chaque anse avec des noms d'esclaves surtout (diverses matrices pour les mêmes noms). On trouve aussi des surnoms et des timbres codés. Un premier examen des pâtes suggère une origine latio-campanienne du chargement. La datation que fournit l'étude des timbres par comparaison avec des trouvailles terrestres indique la fin du $\mathrm{II}^{\mathrm{e}}$ siècle et le premier quart du $\mathrm{I}^{\mathrm{er}}$ siècle avant notre ère. La distribution de timbres identiques en Gaule montre la prédominance de l'axe Aude-Garonne qui n'est au demeurant pas exclusif.

70, L. Long, G. Duperron, « Navigation et commerce dans le delta du Rhône : l'épave Arles-Rhône 14 (III siècle apr. J.-C. ), dans S. Mauné, G. Duperron (éds), Aspects de la vie matérielle en Gaule Narbonnaise II, (I ${ }^{\mathrm{er}}$ siècle av. J.-C. - I $\mathrm{I}^{\mathrm{e}}$ siècle apr. J.-C. ), Montagnac, 2013, p. 125-168, il s'agit d'une embarcation fluvio-maritime de quelque 15 à $20 \mathrm{~m}$ de long dont le chargement témoigne del' activité commerciale variée de la première moitié du III ${ }^{\mathrm{e}}$ siècle. À côté des amphores G. 4, majoritaires, se trouvent des amphores Africaines I et II de l'atelier de Salakta (timbre QCS), des amphores de Lusitanie, Almagro 51C, de Bétique, Beltran 72 et d'Orient. La vaisselle fine et commune vient d'Afrique et de la vallée du Rhône, les lampes sont également africaines et régionales. 


\section{- En Bretagne}

71, A. Fitzpartick, « Republican Amphorae in Iron Age Britain », dans F. Olmer (éd.), Itinéraires des vins romains en Gaule, Monographies d'Archéologie Méditerranéenne $\mathrm{n}^{\circ}$ 5, Lattes, 2013, p. 327-343, présente une mise à jour de la question. Une centaine de sites sont concernés par l'importation de Dr. 1. Plusieurs sites-clés ayant importé des Dr. 1A et des Dr. 1B sont présentés successivement, suivi par ceux concernés par les autres amphores du début de l'Empire. En conclusion, il apparaît que les importations vinaires italiques ne concernent que le sud de l'Angleterre. Quelques gréco-italiques ont été identifiées qui fixent les premières importations à la fin du - $\mathrm{II}^{\mathrm{e}}$ siècle. Les quantités de vins italiques de l'âge du fer en Bretagne sont au demeurant modestes.

\section{- Entre Rhin et Danube}

72, U. Ehmig, «Les traces des dernières Dressel 1 entre Rhin et Danube », dans F. Olmer (éd.), Itinéraires des vins romains en Gaule, Monographies d'Archéologie Méditerranéenne $n^{\circ} 5$, Lattes, 2013, p. 371-375, ce petit article très pertinent montre avec une grande précision combien des villes comme Mayence ou Augst et des camps militaires comme Dangstetten ou Rödgen qui sont tous fondés vers 15 av. J.-C., ou peu après, sont pauvres en Dr. 1. Serait-ce la fin des importations comme le dit A. Desbat à propos de Lyon?

Il n'en est rien, si l'on examine les sites de fondation plus ancienne entre Rhin et Danube qui continuent à importer le vin tyrrhénien, comme le montrent aussi nombre d'épaves et plusieurs autres sites de Gaule que l'on a pu remarquer au cours de ce colloque. S'opposent deux clientèles, l'une nouvelle et l'autre plus ancienne et fidélisée. Au reste, U. E. met en garde sur la confusion possible des lèvres d'amphores Dr. 12 et Dr. 1 d'après les dessins; seul l'examen visuel des tessons permet de les identifier correctement.

73, S. Martin-Kilcher et al., «Les importations d'amphores dans la civitas Rauracorum ( $\mathrm{II}^{\mathrm{e}} / \mathrm{I}^{\mathrm{er}}$ siècle av. J.-C. ) : Bâle-usine à gaz, Sirentz, Sausheim, Bâle-cilline de la cathédrale, Augst », dans F. Olmer (éd.), Itinéraires des vins romains en Gaule, Monographies d'Archéologie Méditerranéenne n5, Lattes, 2013, p. 377-422, le mobilier d'ensembles clos de quatre sites de la Tène finale est pris en compte. Les sites urbains sont mieux fournis en amphores que les sites ruraux, comme généralement. Les importations vinaires italiques arrivent dès la deuxième moitié du $\mathrm{II}^{\mathrm{e}}$ siècle av. notre ère. Quelques amphores gréco-italiques sans doute plus anciennes, sont rares. Des analyses chimiques ont permis de déterminer la provenance des vins d'Italie pendant toute la durée des importations : la Campanie (Mondragone, Minturnes, Pompéi), l'Étrurie centrale 
(Albinia et Cosa), la côte tyrrhénienne et la côte adriatique sans plus de précision, et une part indéterminée. Deux tiers des amphores viennent de grands ateliers connus comme Mondragone, Albinia et Cosa.

\section{En Méditerranée orientale}

\section{- En Asie Mineure}

74, T. Bezeczky, The Amphorae of Roman Ephesus, Vienne, 2013, étudie les amphores de huit sites différents d'Éphèse qu'il décrit, soit 621 amphores souvent fragmentaires. Il met d'abord en évidence par des analyses pétrographiques, 11 types produits à Éphèse ou dans les environs, entre la période hellénistique et la période romaine tardive. Plusieurs d'entre eux imitent des formes connues (égéennes, rhodienne, Kapitän 2, LRA3, monoansées...), tandis que le type 56 semble propre à Éphèse. Vient ensuite l'étude de chacun des 71 types recensés classés chronologiquement, avec leur localisation et datation dans la fouille et une riche étude épigraphique quand il y a lieu. Des analyses pétrographiques sont menées systématiquement, elles permettent parfois d'identifier des groupes de provenances différentes. Des tableaux quantifiés des provenances montrent en particulier que les importations d'Italie et d'Espagne représentent 30\% durant la première moitié du $\mathrm{I}^{\mathrm{er}}$ siècle de notre ère. T. B. nous livre un outil exceptionnel pour une région qui était encore mal connue dans le domaine des amphores.

\section{- En Pamphylie}

75, C. Brixhe, «Timbres amphoriques de Pamphylie », Centre d'Études Alexandrines, 23, 2012, C. B. reprend et complète les travaux de L. Benakis, V. Grace et A. G. Woodhead pour livrer un catalogue de 763 timbres des II $^{\mathrm{e}}$ et $\mathrm{I}^{\text {er }}$ siècle avant notre ère. Les trois quarts d'entre eux sont trouvés à Alexandrie et marquent l'étroite relation qui unit l'Égypte et la Pamphylie. Les proportions d'amphores pamphyliennes à Alexandrie restent cependant modestes par rapport à celles de Rhodes ou Cnide. L'étude des timbres est particulièrement difficile : on ne connait aucun atelier en Pamphylie qui n'a livré au reste que quatre timbres. Aucun des noms des quelque 300 éponymes ou des fabricants mentionnés n'est attribué à une cité. La typologie des amphores est encore mal connue et leur contenu n'est pas très précis. On comprendra pourquoi le catalogue est simplement classé par ordre alphabétique. 


\section{- À Chypre}

76, M. L. Lawall, J. Lund (éds), The transport Amphorae and Trade of Cyprus, Aahrus University Press, 2013, ont réuni 17 courts articles d'auteurs variés sur les amphores produites à Chypre (des plus anciennes comme les amphores à anse de panier, aux plus récentes comme les LR1) et leurs exportations. On trouve également des études sur les amphores trouvées à Chypre et venues d'autres origines, comme les amphores romaines par exemple.

\section{- En Syrie}

77, F. Laubenheimer, «Les amphores », dans A. Schmidt-Colinet, W. Al-As'ad (éds), Palmyras Reichtum durch Weltweiten Handel, Achäologische Untersuchungen im Bereich der Hellenististichen Stadt, Vienne, 2013, vol.2, p. 93-105, les fouilles syro-allemandes du secteur de la ville hellénistique montrent l'ouverture de Palmyre au grand commerce international à toutes les époques. Des amphores sont importées essentiellement pour le vin d'Italie, de Gaule, de Byzacène et de Tripolitaine comme de Méditerranée orientale. La présence d'amphores syriennes en quantité, une découverte nouvelle, laisse la porte ouverte à de nouvelles recherches pour comprendre leur place dans l'économie et la consommation de produits encore non identifiés.

78, F. Laubenheimer, C. Römer Strehl, « Hellenistiche Amphorenstempel», dans A. Schmidt-Colinet, W. Al-As'ad (éds), Palmyras Reichtum durch Weltweiten Handel, Achäologische Untersuchungen im Bereich der Hellenististichen Stadt, Vienne, 2013, vol. 2, p. 106-108, trois timbres sur amphores rhodiennes de la fin du II siècle et du début du Ir siècle avant notre ère soulignent le développement de Palmyre dès la période hellénistique.

\section{AmpHORES TARDIVES}

\section{En Méditerranée occidentale}

\section{- Dans la péninsule lbérique}

79, R. Járrega Domínguez, « Late Roman Amphorae in the Eastern Tarraconensis. Some Chronological and Quantitative Approaches », dans N. Poulou-Papadimitriou, E. Nodarou, V. Kilikoglou (éds.), LRCW, 4, Late Roman Coarse Wares, Cooking Wares and Amphorae in the Mediterranean, BAR International Series, 2616 (I), 2014, depuis 
les études de S. Keay, il y a vingt-cinq ans, de nouveaux contextes bien datés précisent la chronologie et les quantités des différentes importations. La distribution des amphores romaines tardives entre ville et campagne apporte de nouveaux résultats.

\section{- En Afrique}

80, P. Ballet, M. Bonifay, S. Marchand, «Africa vs Aegyptus: routes, rythmes et adaptations de la céramique africaine en Égypte », dans $S$. Guédon (éd.), Entre Afrique et Égypte: relations et échange entre les espaces au sud de la Méditerranée à l'époque romaine, Bordeaux, 2012, p. 87-117, envisagent en parallèle les exportations de céramique et d'amphores africaines vers l'Égypte, sachant qu'il y a en Orient un décalage de plus d'un siècle entre arrivée de sigillée africaines et amphores, d'une part, et que, d'autre part il y a un net découplage dans le transport des vaisselles fines et celui des amphores. Les routes et les origines des divers produits transportés en amphores à travers le temps sont analysées. À côté des routes maritime ou fluviale sont proposées l'alternative de routes terrestres.

81, M. Bonifay, A. Tchernia, «Les réseaux de la céramique africaine ( $\mathrm{I}^{\mathrm{er}}$ $\mathrm{V}^{\mathrm{e}}$ siècles) », dans S. Keay (éd.), Rome, Portus and the Mediterranean, Archaeological Monographs of the British School at Rome, 21, 2012, p. 315-333, les productions et commercialisation de la céramique africaine et celle des amphores sont envisagées en parallèle. Une analyse de la localisation des ateliers et par là une définition plus précise des origines, celles des denrées transportées par les amphores, huile, salsamenta et vin, s'accompagne d'un regard sur les entrepôts, les ports, les bateaux et les marchés. Le commerce continental à l'intérieur de l'Afrique, se différencie du commerce maritime par les produits concernés ou les conteneurs utilisés. La déconnexion de la distribution de la vaisselle et de celle des amphores en Méditerranée est à nouveau soulignée, la première étant plutôt liée au commerce du blé. On s'interroge sur la chute des importations en Méditerranée dans la deuxième moitié du III siècle et au début du $\mathrm{IV}^{\mathrm{e}}$ siècle, puis au $\mathrm{V}^{\mathrm{e}}$ siècle. Les exportations d'amphores à huile, dès la fin du règne d'Hadrien, sont mises en regard avec l'annone et la régularité de l'approvisionnement en huile de la ville de Rome. L'augmentation et la diversification au bénéfice de la Byzacène des importations d'amphores africaines à Rome se fait sentir dès le milieu du $\mathrm{III}^{\mathrm{e}}$ siècle. Les plus forts pourcentages seront atteints à la fin du $\mathrm{IV}^{\mathrm{e}}$ siècle et au début du $\mathrm{V}^{\mathrm{e}}$ siècle. L'huile n'est sans doute pas alors la seule denrée concernée, les arrivages de salsamenta voire du vin sont sans doute accrus. On s'interroge sur la complexité des réseaux qui conduisent à expédier à partir du milieu du III $^{\mathrm{e}}$ siècle des amphores africaines à Cadix pour les redistribuer 
ensuite en Méditerranée. Cet article renouvelle la vision globale et bien documentée du commerce africain telle qu'on peut l'appréhender aujourd'hui.

Signalons aussi, bien que nous n'ayons pas eu les livres en main, la parution en 2013 de l'ouvrage de F. Salviat et A. Tchernia, Vins, vignerons et buveurs de l'Antiquité, L'Erma di Bretschneider, Rome, consacrés aux vins grecs et aux vins romains, et le gros volume (532 p.) de C. Panella et G. Rizzo, Ostia VI. Le terme del Nuotatore I, Studi Miscellanei 38, 2014, «I saggi nell'area » par C. Panella ; «Le anfore. Dinamiche produttive e commerciali nel Mediterraneo di età impériale » par G. Rizzo.

Que soient remerciés tous ceux qui ont bien voulu contribuer à cette chronique en m'adressant des publications. 